\title{
METODOLOGIA ALTERNATIVA DO TESTE DE ENVELHECIMENTO ACELERADO PARA SEMENTES DE CENOURA
}

\author{
Angelica Brod Rodo ${ }^{1,3 *}$; Maristela Panobianco ${ }^{1,4}$; Júlio Marcos Filho 2,4 \\ ${ }^{1}$ Pós-Graduando do Depto. de Produção Vegetal - USP/ESALQ. \\ ${ }_{3}^{2}$ Depto. de Produção Vegetal - USP/ESALQ, C.P. 9 - CEP: 13418-900 - Piracicaba, SP. \\ ${ }^{3}$ Bolsista FAPESP. \\ ${ }^{4}$ Bolsista CNPq. \\ *Autor correspondente <abrodo@carpa.ciagri.usp.br>
}

\begin{abstract}
RESUMO: O presente trabalho teve por objetivo estudar a metodologia do teste de envelhecimento acelerado para avaliação do potencial fisiológico de sementes de cenoura, bem como avaliar a eficiência do uso de solução saturada de sal no controle da absorção de água pelas sementes durante a realização do teste. Para tanto, quatro lotes de sementes de cenoura, cultivar Brasília, foram submetidos aos testes de germinação, emergência das plântulas em casa de vegetação, condutividade elétrica e envelhecimento acelerado $\left(41^{\circ} \mathrm{C}\right.$; 48, 72 e 96h; com e sem o uso de solução saturada de $\mathrm{NaCl}$ ). O teste de envelhecimento acelerado (com e sem sal) permitiu classificar os lotes em diferentes níveis de vigor, de maneira semelhante ao do teste de emergência das plântulas. Diante dos resultados obtidos, pode-se concluir que, dentre os procedimentos adotados para o teste de envelhecimento acelerado, o período de $72 \mathrm{~h}$, com o uso da solução saturada de $\mathrm{NaCl}$, é considerado adequado para avaliação do potencial fisiológico de sementes de cenoura, cultivar Brasília. Observou-se, também, que o grau de umidade das sementes expostas a solução saturada do sal, menor e mais uniforme após os períodos de envelhecimento, revela vantagens na utilização desse procedimento, em relação ao convencional.
\end{abstract}

Palavras-chave: Daucus carota, vigor, potencial fisiológico, germinação

\section{ALTERNATIVE METHODOLOGY FOR THE ACCELERATED AGING TEST FOR CARROT SEEDS}

\begin{abstract}
The objective of the present work was to study the methodology of the accelerated aging test to evaluate the physiological quality of carrot (Daucus carota L.) seeds and the efficiency of using saturated salt solution for water uptake by seeds during the accomplishment of the test. Four lots of carrot seeds cv. Brasília were tested for germination, seedling emergence, electrical conductivity and accelerated aging $\left(41^{\circ} \mathrm{C} ; 48,72\right.$ and $96 \mathrm{~h}$; with and without the use of saturated solution of $\mathrm{NaCl}$ ). Accelerated aging (with and without salt) ranked different vigor levels among seed lots, as well as seedling emergence. It was concluded that, among the accelerated aging protocols studied, the period of $72 \mathrm{~h}$, with the use of the saturated solution of $\mathrm{NaCl}$, is considered appropriate to assess the physiological quality of carrot seeds, cv. Brasília. It was also observed that seed water content after the saturated salt accelerated aging test was lower and more uniform, thus showing advantages in relation to the conventional procedure.

Key words: Daucus carota, vigor, physiological quality, germination
\end{abstract}

\section{INTRODUÇÃO}

A qualidade de um lote de sementes compreende uma série de características ou de atributos que determinam o seu valor para a semeadura, os quais são considerados como de natureza genética, física, fisiológica e sanitária. Destes, pode ser destacado o potencial fisiológico, diretamente responsável pelo desempenho das sementes em campo e armazenamento.

O teste de germinação é utilizado rotineiramente em laboratório, para avaliar o potencial fisiológico das sementes. No entanto, há vários anos, pesquisadores, tecnologistas, produtores de sementes e agricultores não têm se mostrado completamente satisfeitos com as informações fornecidas por esse teste, realizado sob condições de ambiente que geralmente conduzem à superestimativa de sua qualidade. Assim, foram desenvolvidos testes de vigor com o objetivo básico de identificar possíveis diferenças no potencial fisiológico de lotes que apresentem poder germinativo semelhante.

Atualmente, os testes de vigor trazem benefícios a todos os segmentos da produção de grandes culturas e hortaliças. Dentre os testes disponíveis, o envelhecimento acelerado é um dos mais estudados e recomendados para várias espécies cultivadas. Inicialmente desenvolvido com a finalidade de estimar a longevidade de sementes armazenadas (Delouche \& Baskin, 1973), esse teste tem sido amplamente estudado com vistas à sua padronização. Baseia-se no princípio de que lotes de sementes de alto vigor manterão sua viabilidade quando submetidos, durante curtos períodos de tempo, a condições severas de temperatura e umidade relativa em uma câmara apropriada, enquanto que os de baixo vigor terão sua viabilidade reduzida. 
O teste de envelhecimento acelerado, por ser relativamente simples e de fácil execução em laboratório, vem sendo estudado na determinação do vigor de diversas espécies de hortaliças. Trabalhos realizados com cebola (Piana et al., 1995) e cenoura (Martins et al., 1996) demonstraram que, dentre os testes estudados, 0 envelhecimento acelerado foi o que apresentou melhor relação com a emergência das plântulas em campo.

Entretanto, para a maioria das hortaliças e outras espécies com sementes pequenas, o envelhecimento acelerado pode apresentar certas limitações. Sementes pequenas absorvem água mais rapidamente, resultando em deterioração mais acentuada e redução mais drástica da germinação pós-envelhecimento. Para contornar este problema, tem sido sugerida a exposição das sementes a soluções saturadas de sais durante a realização do teste, as quais reduzem a umidade relativa do ambiente no interior dos compartimentos individuais $(\mathrm{NaCl}-76 \% \mathrm{UR}$, $\mathrm{KCl}-87 \% \mathrm{UR}$ ou $\mathrm{NaBr}-55 \% \mathrm{UR}$ ), retardando assim a absorção de água pelas sementes. Este método, denominado teste de envelhecimento acelerado com uso de soluções saturadas de sal (SSAA - "Saturated Salt Accelerated Aging"), foi proposto por Jianhua \& McDonald (1996), os quais, trabalhando com sementes de Impatiens wallerana Hook, verificaram eficiência da solução salina no controle da absorção de água pelas sementes e na avaliação do vigor. Também foi observada maior eficiência do teste de envelhecimento acelerado com uso de soluções saturadas de sal na classificação de lotes de sementes com diferentes níveis de vigor em cultivares de milho doce (Bennett et al., 1998) e de pimentão (Panobianco \& Marcos Filho, 1998).

Diante do exposto, o presente trabalho teve por objetivo estudar a metodologia do teste de envelhecimento acelerado para avaliação do potencial fisiológico de sementes de cenoura, bem como a eficiência do uso de soluções saturadas de sal no controle da absorção de água pelas sementes durante a realização do teste.

\section{MATERIAL E MÉTODOS}

O trabalho foi realizado no Laboratório de Análise de Sementes e no Campo Experimental do Departamento de Produção Vegetal da Escola Superior de Agricultura "Luiz de Queiroz", em Piracicaba - SP, nos meses de abril e maio de 1999.

Foram utilizados quatro lotes de sementes de cenoura, cultivar Brasília, submetidos aos testes descritos a seguir.

a) Determinação do grau de umidade - realizada pelo método da estufa à $105 \pm 3^{\circ} \mathrm{C}$, durante 24 horas, conforme as Regras para Análise de Sementes - RAS (Brasil, 1992).

b) Germinação - conduzido com quatro repetições de 50 sementes para cada lote, semeadas em caixas tipo "gerbox" sobre duas folhas de papel mata-borrão umedecidas com água destilada, na proporção de 2,5 vezes o peso do papel seco, e colocadas para germinar a $20^{\circ} \mathrm{C}$. As contagens foram realizadas aos 7 e 14 dias após a semeadura e as avaliações efetuadas segundo os critérios estabelecidos pelas RAS (Brasil, 1992).

c) Emergência de plântulas em casa de vegetação - foram utilizadas quatro repetições de 50 sementes por lote, semeadas em bandejas de "isopor" com células separadas, contendo substrato comercial, composto por casca de pinus, vermiculita e fertilizante químico (informações obtidas pelo fabricante). As bandejas foram mantidas a $25^{\circ} \mathrm{C}$, em estufa dotada de sistema de nebulização intermitente. As avaliações foram realizadas aos 14 dias após a semeadura, através da contagem de plântulas emergidas com tamanho igual ou superior a $1,0 \mathrm{~cm}$.

d) Condutividade elétrica - foi empregado o método massal, realizado de acordo com a metodologia proposta pelo Comitê de Vigor da International Seed Testing Association (ISTA, 1995). Foram utilizadas quatro repetições de 25 sementes por lote, as quais foram contadas e pesadas e, a seguir, colocadas em copos plásticos, contendo $50 \mathrm{ml}$ de água destilada e embebidas a $25^{\circ} \mathrm{C}$ durante 24 horas. As leituras foram realizadas em condutivímetro Digimed DM-31 e os resultados expressos em $\mu \mathrm{mho} \mathrm{cm}^{-1} \mathrm{~g}^{-1}$ de sementes.

e) Envelhecimento acelerado - foram utilizadas caixas tipo "gerbox", como compartimento individual (minicâmara), possuindo em seu interior uma bandeja com tela de alumínio onde as sementes, após pesagem $(5,0 \mathrm{~g})$, foram distribuídas de maneira a formarem camada uniforme. Dentro de cada compartimento individual foram adicionados $40 \mathrm{ml}$ de água destilada; as caixas foram mantidas em câmara do tipo $\mathrm{BOD}$, a $41^{\circ} \mathrm{C}$, por períodos de 48, 72 e 96 horas. Em seguida, as sementes foram submetidas ao teste de germinação, sendo avaliadas após sete dias e os resultados expressos em percentagem de plântulas normais. Este mesmo teste também foi conduzido empregando-se o procedimento proposto por Jianhua \& McDonald (1996), onde os $40 \mathrm{ml}$ de água destilada adicionados aos "gerbox" foram substituídos por $40 \mathrm{ml}$ de solução saturada de $\mathrm{NaCl}(40 \mathrm{~g}$ do sal/100 ml de água).

O delineamento estatístico utilizado foi o inteiramente casualizado, com quatro repetições. As médias obtidas nas avaliações de cada lote foram comparadas pelo teste de Tukey, a $5 \%$ de probabilidade. Para execução das análises estatísticas foi utilizado o "Sistema de Análise Estatística para Microcomputadores - SANEST" (Zonta \& Machado, 1984). Os dados dos testes de germinação, emergência e envelhecimento acelerado foram transformados em arc sen $\sqrt{x / 100}$, enquanto que os de condutividade elétrica não sofreram transformação. 


\section{RESULTADOS E DISCUSSÃO}

Na TABELA 1, pode-se observar o grau de umidade inicial dos lotes avaliados e o grau de umidade atingido após a realização do teste de envelhecimento acelerado tradicional e com o uso de solução saturada de $\mathrm{NaCl}$. Os resultados demonstraram que o uso de solução saturada de $\mathrm{NaCl}$ promoveu a redução da velocidade de captação de água pelas sementes de cenoura durante 0 período de envelhecimento, de modo que ao final dos períodos estudados as sementes atingiram graus de umidade sensivelmente inferiores aos verificados com o uso do protocolo tradicional; neste caso houve variações mais acentuadas no grau de umidade das sementes ao final do envelhecimento acelerado, confirmando as observações de Jianhua \& McDonald (1996). Desta maneira, as condições de envelhecimento com o uso de solução de sal promoveram efeitos menos drásticos, pois ao atingir menores teores de água, o grau de deterioração das sementes foi atenuado em relação ao normalmente verificado com o uso do método tradicional. Resultados semelhantes foram obtidos em trabalho com sementes de pimentão por Panobianco \& Marcos Filho (1998).

Outro aspecto a ser considerado é que, após o envelhecimento dos lotes, em ambos os métodos estudados, foram observadas diferenças marcantes no desenvolvimento de fungos; no teste de envelhecimento

TABELA 1 - Grau de umidade de quatro lotes de sementes de cenoura, cultivar Brasília, antes e após a exposição ao teste de envelhecimento acelerado (sem sal e com sal). Piracicaba, SP, 1999.

\begin{tabular}{|c|c|c|c|c|c|c|c|}
\hline \multirow[t]{3}{*}{ Lote } & \multirow{3}{*}{$\begin{array}{c}\text { Grau de } \\
\text { umidade } \\
\text { inicial }\end{array}$} & \multicolumn{6}{|c|}{$\begin{array}{l}\text { Grau de umidade após } \\
\text { envelhecimento acelerado }\end{array}$} \\
\hline & & \multicolumn{3}{|c|}{ sem sal } & \multicolumn{3}{|c|}{ com sal } \\
\hline & & $48 \mathrm{~h}$ & $72 \mathrm{~h}$ & $96 \mathrm{~h}$ & $48 \mathrm{~h}$ & $72 \mathrm{~h}$ & $96 \mathrm{~h}$ \\
\hline & \multicolumn{7}{|c|}{ - } \\
\hline 1 & 7,7 & 37,6 & 48,7 & 48,5 & 10,2 & 10,8 & 9,9 \\
\hline 2 & 7,6 & 34,2 & 43,6 & 42,6 & 10,6 & 10,6 & 9,6 \\
\hline 3 & 7,7 & 33,6 & 39,5 & 42,7 & 10,5 & 10,2 & 8,8 \\
\hline 4 & 7,6 & 34,5 & 43,3 & 46,0 & 9,9 & 9,9 & 9,5 \\
\hline
\end{tabular}

com adição de solução de $\mathrm{NaCl}$, praticamente não houve presença de microrganismos. Observação semelhante foi efetuada por Jianhua \& McDonald (1996), pois a baixa umidade relativa no teste de envelhecimento acelerado com adição de solução saturada de sal diminuiu consideravelmente o desenvolvimento de fungos, sendo esta mais uma vantagem dessa metodologia.

Os resultados dos testes de germinação, emergência de plântulas em casa de vegetação, condutividade elétrica e envelhecimento acelerado são apresentados na TABELA 2. Pelos testes de germinação e de emergência de plântulas, considerando-se tanto a análise estatística como o valor prático das comparações entre as médias, o lote 3 foi considerado como de alta qualidade, os lotes 1 e 4 de qualidade intermediária e o lote 2 de baixa qualidade. Com relação ao teste de condutividade elétrica, verificou-se que não houve diferença significativa entre os lotes. Em razão dos altos valores de liberação de eletrólitos, a metodologia adotada na pesquisa provavelmente poderia ser alterada, utilizando-se maior volume de água combinado a uma menor temperatura, visando conferir maior sensibilidade ao teste.

Por outro lado, examinando-se os resultados do teste de envelhecimento acelerado (com e sem sal) conduzidos durante o período de 48 horas, bem como os resultados do envelhecimento acelerado tradicional, após o período de 72 horas, observa-se a mesma classificação dos lotes quanto ao nível de vigor, acompanhando as informações obtidas nos testes de germinação e de emergência de plântulas; assim, o lote 3 apresentou-se como o de mais alta qualidade e o lote 2 como o de qualidade mais baixa, enquanto que os lotes 1 e 4, de qualidade similar entre si, situaram-se em posição intermediária.

Entretanto, os resultados obtidos no teste de envelhecimento acelerado com uso de solução de $\mathrm{NaCl}$, durante 72 horas, demonstraram melhor separação dos lotes em diferentes níveis de vigor, pois além de indicar o lote 3 como o de melhor qualidade e o lote 2 como o de qualidade mais baixa, também detectou diferença entre 0 lote 2 e o lote 1; esta separação não foi verificada na

TABELA 2 - Resultados dos testes de Germinação - G, Emergência de Plântulas- E, Condutividade Elétrica - CE e Envelhecimento Acelerado - EA, sem sal e com sal, em quatro lotes de sementes de cenoura, cultivar Brasília. Piracicaba, SP, 1999.

\begin{tabular}{|c|c|c|c|c|c|c|c|c|c|}
\hline \multirow[t]{2}{*}{ Lote } & \multirow[t]{2}{*}{ G } & \multirow[t]{2}{*}{ E } & \multirow[t]{2}{*}{ CE } & \multicolumn{3}{|c|}{ EA sem sal } & \multicolumn{3}{|c|}{ EA com sal } \\
\hline & & & & $48 \mathrm{~h}$ & $72 \mathrm{~h}$ & $96 \mathrm{~h}$ & $48 \mathrm{~h}$ & $72 \mathrm{~h}$ & $96 \mathrm{~h}$ \\
\hline & \multicolumn{2}{|c|}{----- \% ------ } & $\mu \mathrm{mho} \mathrm{cm}^{-1} \mathrm{~g}^{-1}$ & \multicolumn{6}{|c|}{ - } \\
\hline 1 & $78 a b^{*}$ & $76 a b$ & $1950,5 \mathrm{a}$ & $65 a b$ & $56 \mathrm{ab}$ & $37 \mathrm{c}$ & $73 a b$ & $68 \mathrm{~b}$ & $66 \mathrm{~b}$ \\
\hline 2 & $71 \mathrm{~b}$ & $65 b$ & $1566,3 \mathrm{a}$ & $61 \mathrm{~b}$ & $46 b$ & $34 \mathrm{c}$ & $60 \mathrm{~b}$ & $54 \mathrm{c}$ & $62 b$ \\
\hline 3 & $82 \mathrm{a}$ & 79 a & 1778,4 a & $74 \mathrm{a}$ & $60 a$ & $57 \mathrm{a}$ & $75 a$ & $81 \mathrm{a}$ & $76 \mathrm{a}$ \\
\hline 4 & $75 a b$ & $74 a b$ & $1689,5 \mathrm{a}$ & $67 a b$ & $58 a b$ & $47 \mathrm{~b}$ & $70 a b$ & $60 \mathrm{bc}$ & $65 \mathrm{~b}$ \\
\hline$\overline{\mathrm{CV} \%}$ & 4,7 & 7,2 & 11,8 & 5,1 & 7,6 & 4,8 & 7,0 & 7,3 & 4,0 \\
\hline
\end{tabular}

* Médias seguidas pela mesma letra, na coluna, não diferem pelo teste de Tukey a $5 \%$. 
análise estatística dos dados obtidos nos testes de germinação, de emergência de plântulas e demais procedimentos utilizados para a condução do teste de envelhecimento acelerado. Os resultados dos testes de envelhecimento realizados após o período de 96 horas não foram consistentes, evidenciando somente a melhor qualidade do lote 3 .

Em sementes de cenoura, o período de 72 horas de envelhecimento tem se revelado o mais indicado na classificação de lotes que apresentam diferentes níveis de vigor, em trabalhos de diversos autores (Nascimento \& Andreoli, 1987; Andrade et al., 1995; Trigo \& Trigo, 1995).

Assim, o presente trabalho confirmou que o uso de solução saturada de sal contribui para o aprimoramento da metodologia do teste de envelhecimento acelerado para a avaliação do vigor de sementes pequenas, pois além de utilizar o mesmo equipamento do procedimento tradicional, proporciona condições para absorção de menores quantidades de água e de maneira mais uniforme pelas sementes. Desta forma, esse procedimento alternativo pode auxiliar na padronização do teste de envelhecimento acelerado e permitir que o mesmo seja empregado de maneira eficiente em programas de controle de qualidade de sementes de hortaliças.

\section{CONCLUSÕES}

- O período de 72 horas de envelhecimento, a $41^{\circ} \mathrm{C}$, com o uso de solução saturada de $\mathrm{NaCl}$, é considerado adequado para a avaliação do potencial fisiológico de sementes de cenoura, cultivar Brasília.

- O grau de umidade das sementes expostas a solução saturada de $\mathrm{NaCl}$, menor e mais uniforme após os períodos de envelhecimento, revela vantagens na utilização desse procedimento para sementes pequenas, em relação ao convencional, proporcionando uma taxa de deterioração menos acentuada, resultados menos drásticos e mais uniformes.

\section{REFERÊNCIAS BIBLIOGRÁFICAS}

ANDRADE, R.N.; SANTOS, D.S.B.; SANTOS, B.G.; MELLO, V.D. Correlação entre testes de vigor em sementes de cenoura armazenadas por diferentes períodos. Pesquisa Agropecuária Gaúcha, v.1, p.153-162, 1995.

BENNETT, M.A.; BARR, A.J.; GRASSBAUGH, E.M.; EVANS, A.F. Seed vigor evaluation of su, se and sh2 sweet corn genotypes using the saturated salt accelerated aging (SSAA) test. In: INTERNATIONAL SEED TESTING CONGRESS: SEED SYMPOSIUM, 25., Pretoria, 1998. Abstracts. Pretoria, 1998. p.92-93.

BRASIL. Ministério da Agricultura e Reforma Agrária. Regras para análise de sementes. Brasília, 1992. 365p.

DELOUCHE, J.C.; BASKIN, C.C. Acelerated aging techniques for predicting the relative storability of seed lots. Seed Science and Technology, v.1, p.427-452, 1973.

INTERNATIONAL SEED TESTING ASSOCIATION. Handbook of vigour test methods. 3.ed. Zürich: ISTA, 1995. 117p.

JIANHUA, Z.; McDONALD, M.B. The saturated salt accelerated aging test for small-seeded crops. Seed Science and Technology, v.25, p.123-131, 1996.

MARTINS, L.; SPINOLA, M.C.M.; CALIARI, M.F.; TESSARIOLI NETO, J. Comparação entre métodos para avaliação do vigor de sementes de cenoura (Daucus carota L.). In: SEMINÁRIO PANAMERICANO DE SEMILLAS, 15., Anais. Gramado: ABRATES, 1996. p.11.

NASCIMENTO, W.M.; ANDREOLI, C. Teste de envelhecimento precoce em sementes de cenoura. In: CONGRESSO BRASILEIRO DE SEMENTES, 5., Gramado, 1987. Resumos. Brasília: ABRATES, 1987. p.86.

PANOBIANCO, M.; MARCOS FILHO, J. Comparação entre métodos para avaliação da qualidade fisiológica de sementes de pimentão. Revista Brasileira de Sementes, v.20, p.306-310, 1998.

PIANA, Z.; TILLMANN, M.A.A.; MINAMI, K. Avaliação da qualidade fisiológica de sementes de cebola e sua relação com a produção de mudas vigorosas. Revista Brasileira de Sementes, v.17, p.149-153, 1995.

TRIGO, M.F.O.O.; TRIGO, L.F.N. Determinação da qualidade fisiológica de sementes de cenoura. Informativo ABRATES, v.3, p.134, 1995.

ZONTA, E.P.; MACHADO, A.A. Sistema de análise estatística para microcomputadores-SANEST. Ilha Solteira: UNESP, 1984. 109p.

Recebido em 09.11.99 